\title{
Mathematical Model of Self-Discharge for Supercapacitors
}

www.jestr.org

\author{
Sheryl Dinglasan Fenol ${ }^{1,2, *}$ and Felicito S. Caluyo ${ }^{2}$ \\ ${ }^{1}$ Cavite State University, Cavite, Philippines \\ ${ }^{2}$ Mapúa University, Manila Philippines
}

Received 14 June 2020; Accepted 8 October 2020

\begin{abstract}
Self-discharge is one of the most important considerations in manufacturing supercapacitors. This paper discussed two dynamic mathematical models of self-discharge behavior of supercapacitor from the concept of regular capacitor mechanisms and impedance of an R-C circuit, and constant phase elements (CPE), impedance of R-CPE, and fractionalorder derivatives. The mathematical model was derived from each other to show their equality and relationships.
\end{abstract}

Keywords: Supercapacitor, Self - Discharge, CPE

\section{Introduction}

Self-discharge behavior is an important performance factor in supercapacitors. The term "self-discharge" is sometimes associated with the chemical (faradaic) reactions discharging the surface and excluding any physical processes which cause the voltage drop like charge redistribution [1]. Selfdischarge rates are often higher in supercapacitors compared to batteries which makes self-discharge an important consideration in supercapacitors [2]. The practical supercapacitor electrodes, however suffer from a selfdischarge at the charged state that is caused by leakage currents [3]. This paper discusses two dynamic mathematical models simulating the self-discharge behavior of supercapacitor from different concepts: (1) concept of regular capacitor mechanisms and impedance of an R-C circuit and (2) constant phase elements (CPE), impedance of $\mathrm{R}-\mathrm{CPE}$, and fractional-order derivatives.

\section{Mathematical Background}

\subsection{Capacitor Mechanism}

The equivalent circuit for a regular capacitor can be modelled by representing a capacitor as capacitance $\mathrm{C}$ in parallel with resistance $\mathrm{R}[2]$.

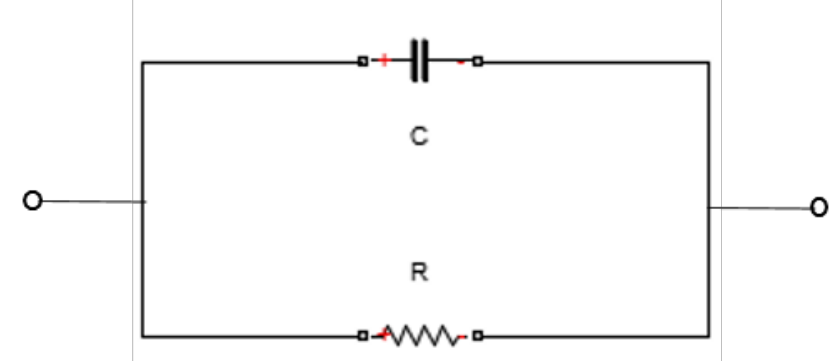

Fig. 1. Equivalent circuit of RC circuit

*E-mail address: sdfenol@crsu.edu.ph

ISSN: 1791-2377 @ 2020 School of Science, IHU. All rights reserved.

doi:10.25103/jestr.135.10
The self-discharge of a regular capacitor can be computed as follows:

$-C \frac{d V}{d t}=i=\frac{V}{R}$

$-C \frac{d V}{V}=\frac{d t}{R}$

$-\frac{d V}{V}=\frac{d t}{R C}$

$-\ln V=\frac{t}{R C}+\tau$

when $t=0, V=V i$

$\ln V_{i}=\tau$

$\ln \left(V_{i} / V_{O}\right)=t / R C$

$V_{i} / V_{O}=\exp (-t / R C)$

\subsection{Fractional Calculus}

The fractional calculus (FC) is the generality of the traditional calculus that leads to similar concepts and tools, but with wider applicability. It allows the derivative and integral operations of arbitrary real or complex order, which makes it suitable for modeling ability.

The general format of fractional linear time-invariant (FLTI) systems equations is as follows:

$\int_{n=0}^{N} a_{n} D^{\alpha_{n}} y(t)=\int_{m=0}^{M} b_{m} D^{\alpha_{m}} x(t)$ when $\alpha_{n}<\alpha_{n}+1$

where $\mathrm{D}$ means derivative and $\alpha_{\mathrm{n}}(\mathrm{n}=0,1,2, \ldots)$ are derivative orders that we will assume to be positive real numbers.

With the use of this definition, the Impulse Response and Transfer Function can be define and compute. Same with other shift-invariant linear system the system described has the exponential as Eigen function. Letting $x(t)=e^{\text {st }}$, where 
$s \in C$ and $t \in R$, we obtain $\mathrm{y}(\mathrm{t})=\mathrm{H}(\mathrm{s}) \mathrm{e}^{\mathrm{st}}$, where $\mathrm{H}(\mathrm{s})$ is the transfer function given by

$H(s)=s^{\alpha}$

Provided that $\mathrm{R}_{\mathrm{e}}(\mathrm{s})>0$ or $\mathrm{R}_{\mathrm{e}}(\mathrm{s})<0$.

With $s=j w$, the Frequency Response, $\mathrm{H}(j w)$ can be obtain, and represents bode diagrams like in usual systems.

The system represented by $\mathrm{s}^{\alpha}$ is called differentiator and Reimann surfaces define one function. Therefore, FLTI equation can represent an infinite number of linear systems. However, only the principal Riemann surface may lead to a real system. Constraining $s^{\alpha}$ by imposing a region of convergence, a transfer function can be defined. Choosing the left half real axis as branch cut line, the transfer function of the usual system can be obtained. Its impulse response is given by

$\delta_{+}^{(\alpha)}(t)=\frac{t^{-\alpha-1} u(t)}{\Gamma(-\alpha)}$

where $\Gamma($.$) is the Euler gamma function.$

With $\alpha=-1$, the normal integrator impulse responses are obtain. Using those impulse responses, the differ integrated of a given signal by the convolution are obtain fractional and led to:

$D_{+}^{(\alpha)} f(t)=\frac{1}{\Gamma(-\alpha)} \int_{-\infty}^{t} f(\tau)(t-\tau)^{-\alpha-1} d \tau$

Although this relation was used for defining fractional derivative this is not convenient analytical point of view. The simplest way of doing it is from the generalized difference which is called forward Grünwald-Letnikov derivative and is given by

$D_{d}^{\alpha} f(z)=\lim _{h \rightarrow 0+} \frac{\sum_{k=0}^{\infty}(-1)^{k}\left(\begin{array}{l}\alpha \\ k\end{array}\right) f(z-k h)}{h^{\alpha}}$

where $\mathrm{h}$ is any complex number in the right hand complex plain [4-10].

\subsection{Constant Phase Element (CPE)}

CPE is a phenomenological term used by Burg in 1984. It is an empirical impedance that its phase angle is independent of frequency. CPE is often related to dispersion attributed to surface inhomogeneities and distributed time constants and obeys Kramers-Kronig relations provided that $|\alpha| \leq 1$.

In electrical engineering, CPE behavior is explained as the frequency dispersion of capacitance by dielectric relaxation, where the electric current density follows the change of an electric field with a delay [11]. Fractional calculus was used to express the phenomenon mathematically. In [12], the model of leaky capacitor was discussed and explained its behavior, the expression for capacitance and current is

$i(t)=C \frac{d^{n} u(t)}{d t^{n}}, \quad 0<n<1$

where $\frac{d^{n} u(t)}{d t^{n}}$ was used as the "fractional-order derivative." The mathematical expression of CPE from fractional derivative and in frequency domain was represented as

$Z_{C P E}=Q s^{-\alpha}$
The coefficient $\mathrm{Q}$ and the fractional exponent $\alpha$ are the parameters of CPE, generally $-1<\alpha<1$ [11-15].

\subsection{Impedance as a Function of Frequency}

Impedance of an electrical element is defined as a function of frequency

$Z(\omega)=\frac{V(\omega)}{I(\omega)}$

where $I(\omega)$ is the electrical response (current) of the element on the applied potential $\mathrm{V}(\omega)$. Impedance can be expressed as a complex quantity when the possible phase shift between current and applied potential were considered.

In polar form

$\bar{Z}(\omega)=|Z(\omega)| \cdot e^{i \theta}$

In rectangular form:

$\bar{Z}(\omega)=Z^{\prime(\omega)}+i Z^{\prime \prime}(\omega)$

The impedance of an electric circuit containing an electrical resistance $\mathrm{R}$ and a capacitor $\mathrm{C}$, in the case of parallel connection is given by

$\frac{1}{\bar{Z}}=\frac{1}{R}+i \omega . \mathrm{C}$

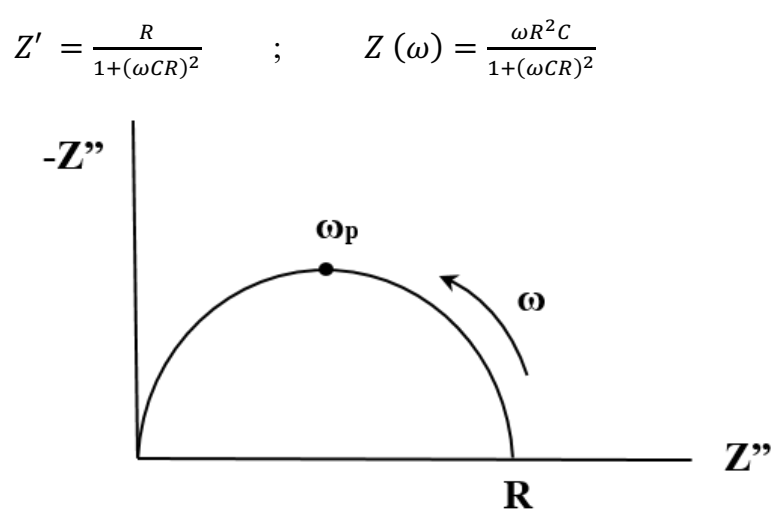

Fig. 2. Impedance semi-circle

Considering Fig. 2, the angular frequency of $\omega$ connects the real and imaginary parts of impedance, the results is a semicircle with diameter equal to the resistance $\mathrm{R}$. The angular frequency at the maximum point of the semicircle defined as $\omega_{\mathrm{p}}$. At this point, the real part of the impedance is equal to its imaginary part, and this equality results in

$\mathrm{C}=\frac{1}{R \omega_{p}}$

ESR is the equivalent series resistance that contributes to the energy los $\mathrm{s}$ during charging and discharging of a supercapacitor. $\mathrm{R}_{\mathrm{P}}$ is the equivalent parallel resistance that simulates energy loss due to supercapacitor self-discharge. Resistor $\mathrm{R}_{\mathrm{P}}$ is always higher than ESR and can be neglected during fast charge/discharge [2].

\section{Self-Discharge Modeling}

This paper proposed two mathematical model for selfdischarge of supercapacitor. The first model was derived 
from the self-discharge characteristics of regular capacitors.

The second model is from the concept of CPE and fractional-order derivatives.

In the first mathematical model the self-discharge is in exponential form. The voltage across the terminal of the supercapacitor is;

$V_{t}(\mathrm{t})=V_{R}(t)+V_{c}(t)$

and the terminal voltage of the supercapacitor in discharging is,

$V_{t}(\mathrm{t})=V_{c}(t)-V_{R}(t)$

The voltage $\mathrm{V}(\mathrm{t})$ can be determined from the equation of parallel RC circuit (see Fig. 1), subject to the initial condition that $\mathrm{V}(0)=\mathrm{V}_{\mathrm{O}}$, the $\mathrm{V}_{\mathrm{C}}(\mathrm{t})$ of can be calculated and expressed as

$V_{c}(\mathrm{t})=V_{o} e^{-t / R_{t} C_{T O T A L}}$

and

$\mathrm{V}(\mathrm{t})=V_{c}(t)+V_{E S R}(t)$

so that,

$\mathrm{V}(\mathrm{t})=V_{O}\left(1+\frac{E S R}{R_{P}}\right) e^{-t / R_{t} C_{T O T A L}}$

Converting the equation into self-discharge and adding exponent " $n$ " to the time constant $1 / \mathrm{RC}$ to consider a fractional capacitor.

$V_{S C}(t) / V_{o}=* \exp -\left[\left(t-t_{o} / R_{p} C\right]^{1-n}\right.$

$V_{S C}(t)=V_{o} *\left(1+\frac{E S R}{R_{P}}\right) * \exp -\left[\left(t-t_{o} / R_{p} C\right]^{1-n}\right.$

Since $1+\frac{E S R}{R_{P}}$ is almost equal to 1 , the simplified mathematical equation is

$V_{S C}(t)=V_{o} * \exp -\left[\left(t-t_{o} / R_{p} C\right]^{1-n}\right.$

In last equation above, $V_{S C}(t)$ is the voltage during selfdischarge, $V_{O}$ is the maximum voltage after charge, $t$ is the time of self-discharge, $t_{O}$ is the charging time, $R_{p}$ is the leakage resistance, and $C$ is the maximum capacitance.

To compute for value of $n$

$\ln \left(V_{C} / V_{S C}\right)=\exp \left(t / R_{p} C\right)^{1-n}$

$1-n=\frac{\ln \left[\ln \left(V_{C} / V_{S C}\right)\right]}{\ln \left(t / R_{p} C\right)}$

where $V_{C}$ is the maximum charged voltage, $V_{S C}$ is the selfdischarge voltage, $t$ is the time of self-discharge $R_{p}$ is the leakage resistance, and $C$ is the rated capacitance.

In second mathematical model, fractional-order derivative was used to convert an impedance formula in frequency domain for R-CPE circuit.

The formula to compute the total impedance is

$$
Z=i \omega L+R_{s}+\sqrt{\frac{R_{e}}{(i \omega)^{d} C}} \cdot \operatorname{coth} \sqrt{(j \omega)^{d} R_{e} C}
$$

and when $\omega$ is equal to infinity

$Z \cong \sqrt{\frac{R_{e}}{(i \omega)^{\alpha} C}} \quad(\omega \rightarrow \infty)$

but when $\omega$ is equal to zero

$Z \cong R_{s}+R_{e}+\frac{1}{(i \omega)^{\alpha} C} \quad(\omega \rightarrow 0)$

CPE can be computed as

$Z_{C P E}=\frac{1}{(i \omega)^{\alpha} C}$

and

$\alpha=1-\gamma$

Using fractional order and laplace transform in solving for the order of the system $\alpha$.

$Z(S)=\frac{1}{C S^{(1-\gamma)}}$

$\mathcal{L}^{-1} Z(s)=\frac{1}{C} \mathcal{L}^{-1} \frac{1}{S^{(1-\gamma)}} 1$

The time domain of $\mathrm{Z}_{\mathrm{CPE}}$ is now

$Z(t)=\frac{1}{C} \frac{t^{1-\gamma}}{\Gamma(2-\gamma)} \mathrm{t}$

Transforming the formula from impedance to voltage

$V(t)=I_{c} \times\left(R_{s}+R_{e}+\frac{t^{1-\gamma}}{\mathrm{C} . \Gamma(2-\gamma)}\right)$

$V(t)=I_{c} \times\left(R_{s}+R_{e}+\frac{t^{1-\gamma}}{\mathrm{C} . \Gamma(2-\gamma)}\right)-I_{c} \times\left(R_{s}+R_{e}+\right.$

$\left.\frac{(t-t c u t-o f f)^{1-\gamma}}{\text { С. }(2-\gamma)}\right)$

where ESR and $V_{O}$

$E S R=R_{s}+R_{e}$

$V_{o}=I_{c} \times E S R$

and the equation for charging and self-discharge are

$V(t)=V_{o}+I_{c} \frac{t^{1-\gamma}}{\text { C. }(2-\gamma) !} \quad$ - charging

$V(t)=V_{o}+I_{c}\left(\frac{t^{1-\gamma}-(t-t c u t-o f f)^{1-\gamma}}{\mathrm{C}(2-\gamma) !}\right) \quad-$ self-discharge $(43)$

where $V(t)$ is the voltage self-discharge, $V_{O}$ is the initial voltage before self-discharge, $I_{C}$ is the charging current, $t$ as the self-discharge time and $C$ is the rated capacitance.

\section{Derivation of self-discharge mathematical model}

The concept of complex number conversion from rectangular to polar form can be applied in both potential and impedance to prove their equality.

$Z=|Z(\omega)| \cdot e^{i \theta} \quad($ polar form $)$ 
$Z=Z^{\prime}(\omega)+i Z^{\prime \prime}(\omega) \quad$ (rectangular form)

The two mathematical model of self-discharge is almost equal to each other,

$V_{S C}(t)=V_{o}+I_{C}\left(\frac{t^{1-\gamma}-\left(t-t_{c u t-o f f}\right)^{1-\gamma}}{\mathrm{C}(2-\gamma) !}\right) \quad \approx \quad V_{S C}(t)=$
$V_{o} * \exp -\left[\left(t-t_{o} / R_{p} C\right]^{1-n}\right.$

equation in the left side is in rectangular form while equation at the right side is in polar form.

First, consider equation at the left side where

$V_{o}=I_{C} x E S R$

while

$\left(\frac{t^{1-\gamma}-\left(t-t_{c u t-o f f}\right)^{1-\gamma}}{\mathrm{C}(2-\gamma) !}\right)=Z(t)$

SO

$V_{S C}(t)=V_{o}+V(t)$

$V_{O}$ is real part of the potential while $V(t)$ is the imaginary part of the potential.

Then, consider equation at the right side where $V_{O}$ is the magnitude and

$\exp -\left[\left(t-t_{o} / R_{p} C\right]^{n}\right.$

is the angle in polar form.

However, $\gamma$ is almost equal to $n$, where $\gamma$ came from $\alpha$ (CPE) with equation

$\alpha=1-\gamma$

and to prove the relationship between $n$ and $\alpha$, we can use the parallel R-CPE and RC circuit and plot.

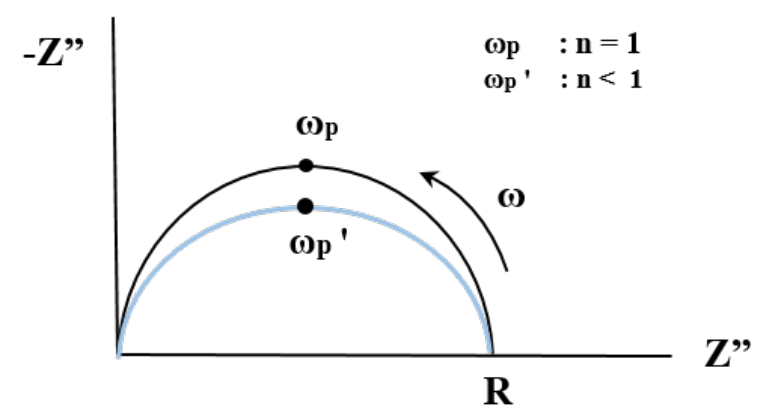

Fig. 3. Complex-plane impedance plot with $n=1$ and $n<1$

In R-CPE, the total impedance is

$Z=\left(\frac{1}{R}+\frac{1}{z_{C P E}}\right)^{-1}$

The impedance of $\mathrm{CPE}\left(\mathrm{Z}_{\mathrm{CPE}}\right)$ is

$\frac{1}{Z_{C P E}}=Q(i \omega)^{\alpha}$

where $Q$ is a CPE parameter that represents a capacity $C$.

However, in an R-C circuit the total impedance is

$$
Z=\frac{1}{R}+i \omega . C
$$
as

The real and imaginary part of impedance can be written

$$
Z^{\prime}=\frac{R}{1+(\omega C R)^{2}} \quad ; \quad Z(\omega)=\frac{\omega R^{2} C}{1+(\omega C R)^{2}}
$$

In Fig. 3 where $n=1$, the real part of impedance equal to its imaginary part and produce an equation of

$$
\mathrm{C}=\frac{1}{R \omega_{p}}
$$

so

$\omega_{p}=\frac{1}{R C}$

while in R-CPE

$\omega_{p}^{\prime}=\frac{1}{(R Q)^{1 / n}}$

Assuming that $\omega_{\mathrm{p}}$ and $\omega_{\mathrm{p}}$ ' for both parallel R-CPE and $\mathrm{R}-\mathrm{C}$ circuits (fig. $\mathrm{n}$ ) are equivalent

$\frac{1}{R C}=\frac{1}{(R Q)^{1 / n}}$

To calculate 'real' capacity $C$ :

$C=R^{\frac{1-n}{n}} \cdot Q^{\frac{1}{n}}$

$Q^{\frac{1}{n}}=\frac{C}{R^{\frac{1-n}{n}}}$

Raised both sides by $n$ :

$Q=\frac{C^{n}}{R^{1-n}}$

$Q=C^{n} R^{n} R^{-1}$

$\frac{1}{Z c p e}=\frac{(R C)^{n}(i \omega)^{n}}{R}$

and if the last equation above $Z_{C P E}$ can be computed using a real capacity $C$ and resistance where $n$ is equal to $\alpha$.

\section{Mathematical Model vs. Actual Simulation}

Three commercially available supercapacitors were used in the actual simulation using 580 Battery Test System and Bplot software. Both supercapacitors were charge with constant current of $1 \mathrm{~mA}$ and held in constant voltage of $2.5 \mathrm{~V}$ for two hours.

Table 1. Manufacturer's given parameters

\begin{tabular}{l|c|c|c}
\hline \multicolumn{1}{c|}{ Component } & A & B & C \\
\hline Rated Capacitance (F) & 3.3 & 4.7 & 10 \\
Rated Voltage (V) & 2.5 & 2.5 & 2.5 \\
Temperature Range $\left({ }^{\circ} \mathrm{C}\right)$ & -25 to 60 & -26 to 60 & -40 to 70 \\
\hline
\end{tabular}


Sheryl Dinglasan Fenol and Felicito $S$. Caluyo/

Journal of Engineering Science and Technology Review 13 (5) (2020) 77 - 81

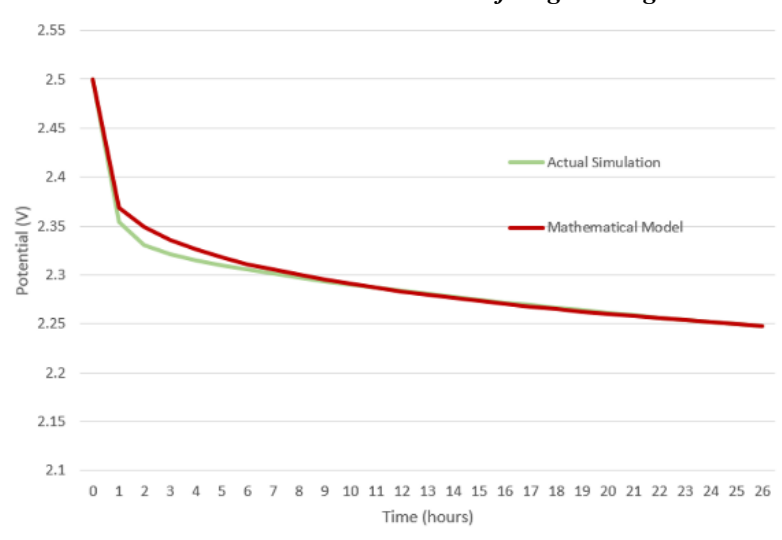

Fig. 4. Simulation Result of Component A

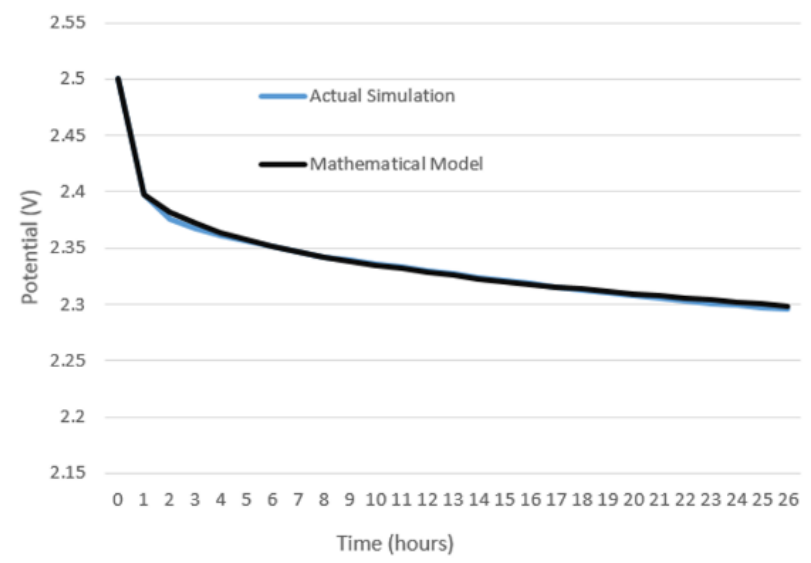

Fig. 5. Simulation Result of Component B

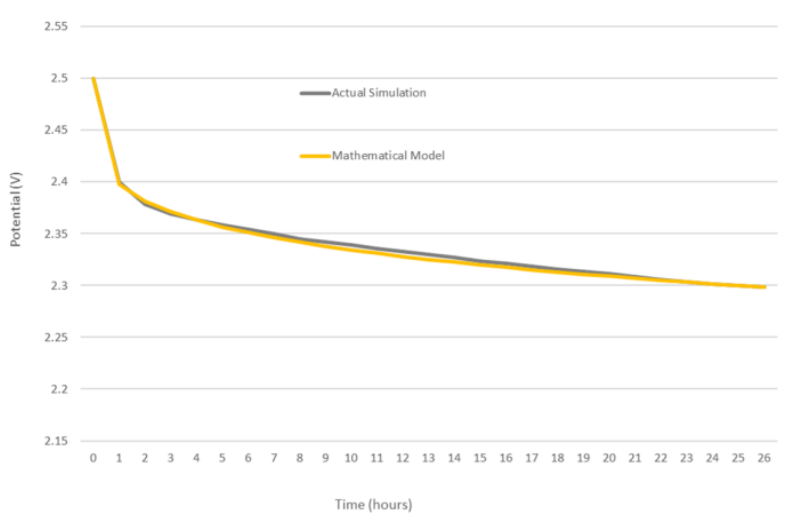

Fig. 6. Simulation Result of Component C

The first mathematical model was used to compare with the actual simulation done in potentiostat since both model and experiment is in time domain. The mathematical model is in good agreement with the actual simulation results as seen in Fig. 4, Fig. 5 and Fig 6. Table II shows the computed and extracted parameters from the mathematical model and actual simulation.

Table 2. Extracted parameters

\begin{tabular}{l|c|c|c}
\hline \multicolumn{1}{c}{ Component } & A & B & C \\
\hline$n$ & 0.208 & 0.215 & 0.215 \\
ESR $(\Omega)$ & $2.028 \times 10^{-03}$ & $3.168 \times 10^{-04}$ & $4.000 \times 10^{-02}$ \\
Leakage Current $(\mathrm{A})$ & $6.692 \times 10^{-06}$ & $4.467 \times 10^{-06}$ & $3.125 \times 10^{-02}$ \\
\hline
\end{tabular}

\section{Acknowledgement}

Supported by Engineering Research and Development for Technology (ERDT) of Department of Science and Technology (DOST) Philippines. Authors would like to acknowledge Commission on Higher Education (CHED), Dr. Wataru Sugimoto and Sugimoto laboratory members, and Shinshu University, Nagano, Japan for help and support.

This is an Open Access article distributed under the terms of the Creative Commons Attribution License

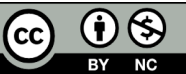

\section{References}

1. H. A. Andreas, "Self-Discharge in Electrochemical Capacitors: A perspective Article," Journal of The Electrochemical Society, vol. 162, no. 5, pp. A5047-A5053, 2015.

2. B. E. Conway, Electrochemical Supercapacitors: Scientific Fundamentals and Technological Applications, New York: Kluwer Academic, 1999.

3. Jong H. Jang, "Complex Capacitance Analysis on Leakage Current Appearing in Electric Double-Layer Capacitor Carbon Electrode," Journal of the Electrochemical Society, vol. 152, no. 7, pp. A1418A1422, 2005.

4. J. Lorenzo, "Parameter Extraction Method for Fractional Order Supercapacitor System Model," in IEEE-ICSE, Kuala Lumpur, Malaysia, 2016.

5. Valeriy Martynyuk, "Fractional model of an electrochemical capacitor," Signal Processing, vol. 107, pp. 355-360, 2015.

6. R. Magin, "On the fractional signals and systems," Signal Processing, vol. 91, pp. 350-371, 2011.

7. S. Das, Functional Fractional Calculus for System Identification and Controls, London: Springer, 2008.
8. M. Ortigueirra, Fractional Calculus for Scientist and Engineers, New York: Dordrecht, 2011.

9. V. Uchaikin, Fractional Derivatives for Physicist and Engineers, Berlin: Springer, 2013.

10. H A. Kilbas, Theory and Applications of Fractional Differential equations, Amsterdam: Elsevier, 2006.

11. M. Itagaki, "Deviation of capacitive and inductive loops in the electrochemical impedance of the dissolving iron electrode," Japan Society of analyticala Chemistry, vol. 18, pp. 641-644, 2002.

12. S. Westerland, "Capacitor Theory," IEEE Transactions on Dielectric and Electric Insulation, vol. 1, pp. 826-839, 1994.

13. P. Zoltowski, "On the electrical capacitance of interfaces exhibiting constant phase element behavior.," Journal of Electroanalytical Chemistry, vol. 443, pp. 149-154, 1998.

14. Shoar Abouzari, "On the Physical interpretation of constant phase elements," Solid State Ionics, vol. 180, pp. 922-927, 2009.

15. K. Biswas, "Realization of a Constant Phase Element and Its Performance Study in a Differentiator Circuit," IEEE Transactions on Circuits and Systems, vol. 53, no. 9, pp. 802-806, 2006. 